\title{
Acceptability of Intermittent Screening and Treatment (IST) versus Intermittent Preventive Treatment (IPT) for the control of malaria in pregnancy: perspective of the Nigerian pregnant woman
}

\author{
Danlami W. DAYOM ${ }^{1,2 *}$, Ehijie F.O. ENATO ${ }^{2}$, Godwin P. EKPE ${ }^{2,3}$ and Ibrahim A. \\ KAMAL ${ }^{2,4}$ \\ ${ }^{l}$ Department of Clinical Pharmacy and Pharmacy Practice, University of Jos, Nigeria. ${ }^{2}$ Department of Clinical \\ Pharmacy and Pharmacy Practice, University of Benin, Benin City. Nigeria. ${ }^{3}$ Department of Clinical Pharmacy \\ and Biopharmacy, University of Uyo. Nigeria. ${ }^{4}$ Pharmacy Department, Federal Medical Centre, Keffi. Nigeria.
}

Received 30 $0^{\text {th }}$ March 2020; Accepted $3^{\text {rd }}$ September 2020

\begin{abstract}
This study qualitatively compared the acceptability of intermittent preventive therapy with an alternative intervention - intermittent screening and treatment for prevention of malaria in pregnancy (MiP) among postpartum women in Edo State, Nigeria. Four focused group discussions were held with postpartum women who participated in a multi-center clinical trial that compared intermittent preventive therapy and intermittent screening and treatment for malaria in pregnancy between 2014 and 2015. The focus group discussions were guided by semi-structured open ended questions covering topics related to their experiences and choice of either interventions. Discussions were analyzed inductively based on emerged themes. Intermittent screening and treatment was most preferred and acceptable by the study participants compared to the intermittent preventive treatment approached. The quest to know their health status through the investigations was a motivation for their choice of the intervention. The rejection of intermittent preventive therapy was due to the general fear of medication use during pregnancy without apparent indication considering the side effects experienced with SP-based intermittent preventive therapy by women who considered themselves healthy. A properly designed and implemented intermittent screening and treatment programme could therefore be more effective in reducing the burden of malaria in pregnancy in the country.
\end{abstract}

Keywords: Acceptability; Focus group discussions; Malaria prevention; Pregnant women

\section{INTRODUCTION}

Though preventable, treatable and curable, malaria remains a major public health problem in Nigeria, taking its greatest toll on children less than five years and pregnant women [1]. In endemic countries, pregnant women have a higher risk of malaria disease due to their physiological and hormonal changes and reduced immunity compared to other adults. In areas of stable, moderate to intense transmission such as Nigeria, Plasmodium falciparum infection in pregnancy occurs without symptoms, yet it is an important contributor to maternal morbidity, preterm delivery, perinatal morbidity and mortality $[2,3]$. These adverse

*Correspondence. E-mail: dayomd123@yahoo.com Tel: +234-8036144149.

ISSN 0189-8442

(cc) BY-No 202. Published by Faculty of Pharmaceutical Sciences, University of Jos, Nigeria. Under Creative Commons Attribution-NonCommercial 4.0 International License. https://creativecommons.org/licenses/by-nc/4.0/ 
outcomes are more frequent in primigravidae [4]. Malaria in pregnancy has been reported to be associated with an increased risk of postpartum hemorrhage [5].

Intermittent preventive treatment (IPT) using sulphadoxine/pyrimethamine (SP) is currently one of the several interventions for preventing malaria in pregnancy $(\mathrm{MiP})$ in Nigeria as recommended by the World Health Organization (WHO). Sulphadoxine/ Pyrimethamine has enjoyed long period of usage for IPT because of its ease of administration and long half-life. However, its current use is threatened by the growing reports of resistance of plasmodium parasite [6] and this has questioned its future effectiveness [7]. Several alternative drugs have been tried as replacements [8-10] but have been largely unsuccessful and so an alternative preventive approach is required altogether. Intermittent screening and treatment (IST) is being considered to be that alternative approach if found to be superior to IPT.

Intermittent screening for malaria infection using a malaria rapid diagnostic test (RDT) at scheduled ANC visits and treating those who test positive to RDT with appropriate antimalarial drug (example, artemisinin-based combination therapy (ACT)) combined with an effective vector control programme is envisaged to provide a potential alternative to SP-IPT[11]. What is needed is to improve on the sensitivity of RDT technology to detect subclinical and recrudescence malaria. This strategy is supported by the fact that malaria transmission in Nigeria is largely seasonal and there is global decline in the burden of malaria in general [12] as it is reported that between 2000 and 2015, 17 countries successfully eliminated malaria and an additional 10 countries were expected to eliminate malaria by 2020 [13]. Based on these successes, malaria eradication is being explored as a feasible global goal [14].
Reports have also shown that there are many factors that have the potential to influence the implementation of health interventions to include: the complexity of the intervention [15]; the capacity of the health system and healthcare providers to deliver the interventions at the scale required; and the acceptability of the interventions to providers and users [16]. Here, the views and opinions of participants in an SP-IPTp verse AL-ISTp clinical trial were assessed using focus group discussions with the aim of assessing their acceptability of either AL-IST or SP-IPT for malaria control and prevention in pregnancy in Nigeria.

\section{METHODS}

Ethical issues. Ethical clearance was obtained from the Research Ethics Committee of University of Benin Teaching Hospital (UBTH) (ref. number: ADM/E 22/A/VOL.VII/946 dated 11/9/2013) as part of a multicenter randomized controlled trial prior to commencement of the study. Administrative approvals were obtained from authorities of study facilities and units. Written informed consent was obtained from all women who were part of the controlled trial and met the inclusion criteria.

Study areas. The study was conducted between February 2014 and September, 2015, in Referral Medical Centre (RMC), Afuze; General Hospital, Afuze; Sure-P Clinic, Otuo; Sure-P Clinic, Ugbekun, and Central Hospital, Benin City, Edo State, Nigeria. The RMC Afuze, General Hospital, Afuze, and Sure-P Clinic, Otuo, are located in the rural parts of the state, while Sure-P Clinic, Ugbekun, and Central Hospital are located in Benin City (State capital).

Study population and sampling. Women who attended antenatal clinic at the study facilities and participated in a two-arm randomized clinical trial and whose complete pregnancy outcomes were available. 
Women were sampled purposively for discussion from among study participants with complete pregnancy outcomes of interests who were part of the two-arm randomized controlled trial conducted in the study areas to compare the efficacy and acceptability of SPIPT and IST in reducing the risks and consequences of MiP in which Women in the IST arm were screened with malaria RDT at enrolment, between 16 and 32 weeks of gestation and at subsequent visits (at least one month from date of enrolment). Only women with positive malaria parasite tests were treated with Artemether-Lumefantrine (Coartem $\left.{ }^{\circledR}\right)$, while those with negative test results were not given any form of antimalarial drug treatment. Women in the SP-IPT arm were given SP at scheduled intervals in the second and third trimesters as recommended by the WHO; however, those who had malaria at any time during the study were treated according to the standard treatment guidelines as recommended by the national malaria treatment guideline. Women were selected based on their possessing specific characteristics according to the description below as presented in figure 1 .

Sample size. Pregnancy outcomes were categorized into four, based on some specific criteria of interest and cutting across all strata of the experiences gathered during the antenatal clinic in addition to pregnancy. Ten women from each category were invited for the discussion totaling forty (40) women

Data collection. Four (4) focus group discussions were held. Discussion questions were carefully structured to enable discussants to freely express their experiences, perceptions, opinions and expectations regarding the two interventions and to assess which intervention they preferred most. Women were grouped based on their clinical test results and delivery outcomes (Figure 1).

Women were so selected to have representation from the treatment arms, age groups and gravidity (Table 1). This was necessary in order to have all the varied experiences in the study captured in the FGD. Group $1\left(\mathrm{FG}_{1} \mathrm{P}\right)$ were those in the SP-IPT arm who took the 2 doses of SP as recommended, did not have third trimester anemia during pregnancy and delivered children with normal birth weights. Group $2\left(\mathrm{FG}_{2} \mathrm{X}\right)$ were those in the IST group who never tested positive for malaria, had no third trimester anaemia and delivered children with normal birth weights. Group $3\left(\mathrm{FG}_{3} \mathrm{C}\right)$ were women in the IST arm who tested positive for malaria parasite at either first, second or both contacts irrespective of delivery outcomes or other clinical data, and group $4\left(\mathrm{FG}_{4} \mathrm{~A}\right)$ were women who had third trimester anemia and or delivered children with low birth weights irrespective of their study arm (Table 1).

Women were excluded from the discussion if they had still birth, fetal abnormalities, delivered away from the study facility, their babies died after discharge from maternity, they declined invitation to participate. Women who had still birth and fetal abnormalities were excluded in the discussions because these adverse outcomes may not entirely be the result of their exposure to the study protocols and this can affect their objective assessment of their preference for either interventions. Ten to fifteen women were randomly selected from each group and contacted on phone to participate in the discussion. Those who consented were listed as participants and were reminded through phone calls and text messages on the date, time and venue for the FGDs. The questions that guided the FGD was designed to elicit their ANC experiences, tests, preference for either interventions, and what motivated them to consent to be tested during their last ANCs. The discussions were moderated by one of the authors (DWD), two authors took notes from the discussions and another tape-recorded using a hand-held Sony recorder. Names and identity of the study participants were coded according to their groups and were identified 
by the codes throughout the discussion and in transcript notes. All discussions were conducted in English. The recorded discussions were played-back, transcribed and analyzed alongside the written notes according to themes that emerged from the discussions. Inductive method was used for the analysis of the audio textual data collected.

\section{RESULTS}

Major themes that emerged from the discussions were identified to be: (i) Choice and reasons for the choice of one intervention over the other compared in the study, (ii) General feelings and experiences on the repeated tests, test results and implications (iii) experiences with drugs taken during pregnancy (iv) Interesting experiences of ANC attendance in general and the last ANC in particular (v) Benefits of ANC services as motivations for attendance by pregnant women and (vi) suggestions for improvement in ANC services rendered to pregnant women.

Choice and reasons for the choice of specific intervention. On their choice for a specific intervention, most women in all the groups recounted their last and earlier ANC experiences to support their choices. To study participants gave reasons for their choice of intervention, which were completely unrelated to their clinic experiences. One participant observed that the two interventions should be combined, that is, if the malaria test is negative, the women should receive SP-IPT.

For some of the reasons presented below majority of the study participants preferred IST to IPT. Some switched preference for the interventions when other discussants advanced "convincing" reasons why they chose a particular intervention suggesting that some choices were made merely on the opportunity given to them to choose without looking intently into the advantages and disadvantages of the two interventions. However, it was common place to find some women sticking to their choices based on what they believe were good for them. These are excerpts of their submissions: 'I prefer both IST and IPT, when they test and you don't have malaria, they should give you the Fansidar for prevention. Like myself now, all the tests you did for me it was all negative but I know that I have malaria, so iff

Fansidar is taken, it prevents the malaria' $\left(\mathrm{FG}_{4}\right.$ woman $\mathrm{A}_{3}$ ).

'I prefer to be tested to know if I have malaria or not, and if I do not have, no need for the Fansidar' $\left(\mathrm{FG}_{4}\right.$ woman $\left.\mathrm{A}_{5}\right)$. 'I prefer to be tested to know if I have malaria or not, if it is confirmed that I have malaria, then you treat me and my baby. But, if you say you want to take Fansidar, I don't really know about that' $\left(\mathrm{FG}_{1}\right.$ woman $\left.\mathrm{P}_{5}\right)$. 'I prefer taking the Fansidar ${ }^{\circledR}$ to prevent the malaria from coming since I know that I am pregnant, I feel I should prevent it than to wait' to have the malaria $\left(\mathrm{FG}_{1}\right.$, woman $\left.\mathrm{P}_{14}\right)$.

'I prefer that preventive medicine, because by the time you prevent it, it won't attack you, they say prevention is better than cure, by the time you are infected before you start taking drug, what if the malaria does not go in time and you are pregnant of a baby, malaria can even destroy the baby, so it is better to prevent it by taking Fansidar ${ }^{\circledR}$ so that you will not be attacked by malaria' ( $\mathrm{FG}_{3}$ woman $\mathrm{C}_{8}$ ).

These responses demonstrate that most of the women do not understand the underlying principle behind the SP-IPT policy. They view the word preventive in light of immunization which is not the case with IPT. However, the understanding and experiences of other discussants shed more light on it, as shown below:

'I prefer doing the test before taking the drug because a pregnant woman is not supposed to take drug anyhow, so I prefer to be diagnosed first, if I have malaria, I go for treatment. On the other hand, if I do not have it there is no need taking the drug. Secondly, malaria and immunization are two different issues, Fansidar does not prevent like immunization because the pregnancy is long, 9 months, you may take Fansidar and be free for a month and later you are infected with malaria at the later part of the pregnancy and so Fansidar cannot be used to compare with immunization we give to children' $\left(\mathrm{FG}_{2}\right.$ woman $\left.\mathrm{X}_{2}\right)$.

'Do not think that Fansidar is that powerful to take care of me throughout the pregnancy. I see 
Fansidar just like Paracetamol and it is not always advisable in the first trimester., I will use myself as an example, in my first pregnancy, I did not take any drug until I was 8 months when I complaint of bitter taste, the doctor wrote that 4,4 morning and evening medicines which they gave me in the pharmacy. When I got home, I started taking it, but I did not finish taking the medicine when I stopped feeling the kick of the baby, that was how I lost that baby. After that, my subsequent pregnancies, I didn't take drugs; I now fear drugs because of that first experience. That Fansidar, I am now afraid of it because if they say take the three at once, I do take it may be one tablet today, after some other days'... (participants cut in with laughter) $\left(\mathrm{FG}_{2}\right.$ woman $\mathrm{X}_{8}$ ).

General feelings and experiences on the repeated tests, results and implications. It was generally mixed reactions; feeling of joy, fear and sadness expressed by the women having to run those tests repeatedly during the ANC. Feeling of joy, because of the benefit of knowing their health status especially when the results were on the desirable side, but feeling of fear because of the pains associated with the test and sadness when the test results were not good enough, implying they are at risk. The motivation to know their health status helped them to endure and bear the pains of pricking their finger-tips to collect blood. When asked how they felt when tested and told their PCV was low or they had malaria, woman $\mathrm{A}_{12}$ in $\mathrm{FG}_{4}$ said:

"I was really afraid because I knew it was nearing time for me to deliver, you said it was $28 \%$ (PCV), I said where will I boost it, when will I eat again to boost it within this short time about to deliver. Such that when I was told I was in labor, I said I cannot be in labor because I remember that my PCV was low'.

'Some of the tests make you sad, some make you happy but I was happy I did it. I never knew my PCV was low because I was feeling okay until you checked me and told me it was low (21\%), I doubted it at first but you told me how to boost it and I did, my second test was 29\%, I was happy, $\left(F_{3}\right.$ woman $\left.C_{6}\right)$.

'I do not like the aspect of pricking to take blood for fear of needle but for the ANC, I like the PCV tests very well because it helps monitor one's blood level and making effort to boost it whenever it is low. If you can do it every clinic visit till delivery, it will be okay' $\left(F G_{2}\right.$, woman $\left.X_{10}\right)$.

Perception with drug taking during pregnancy. Fear and apprehension about drug taking during pregnancy is common occurrence across women of all parities. It takes reassurance to convince women who have had experiences such as this to take any drug without convincing indication for it. Besides this, many pregnant women consider 3 tablets of Fansidar as too powerful for them.

'Test and treat' is better because for me, I don't like drugs. If I am not feeling fine, I should know why and if you test me and I don't have malaria, you want me to prevent if by taking drugs, I am not going to take it, but if you test me and tell me that I have malaria, I will bear it, close my eye and swallow the drug' $\left(\mathrm{FG}_{3}\right.$ woman $\mathrm{C}_{7}$ ).

Complains of side effects of SP such as nausea, vomiting, diarrhea, dizziness and body weakness are commonly reported as a barrier to the choice of IPT by pregnant women [17]. Presence of malaria is therefore a motivation for taking antimalarial drug for this category of people. These comments and observations are indications of the preference of IST over IPT by the discussants, advancing convincing disadvantages and experiences inherent with the IPT implementation. However, women expressed confidence in the safety and benefit of haematinic (routine drugs) and paracetamol use during pregnancy without any fear.

Interesting experiences of the last ANC. The women expressed distinct experiences in their last ANC compared with earlier ANCs because of the free PVC and malaria tests which they never had before. Other interesting experiences of the last ANC included: women who came for ANC and were eventually admitted and delivered same day, spiritual upliftment and health topics taught at every antenatal visit.

'Most interesting was the last PCV test you did for me before my delivery, it came up, because 
it was low before. You told me what to do and I did them and I saw that my PCV came up again. This time I was happy that I did the test' $\left(F G_{3}\right.$, woman $\left.C_{11}\right)$.

My first baby, before I gave birth, I was always down with malaria, when I went to clinic, they prescribed one medicine for me but the pharmacist said 'how can they prescribe this drug for you?! He told me not to take it, I was scared, but this one, I was lucky to meet you on time, I had malaria and you gave me that Coartem free and since then, till my delivery, I did not experience anything, I felt I had cured it with Coartem ${ }^{\circledR}$ ' ( $F G_{3}$ woman $\left.C_{8}\right)$.

The nurses teach, pray, and even when people come from home with problem, they get over it quickly. Secondly, your own aspect of it, I think the free tests, free malaria medicine and things like that which in other places and time are paid for was a very interesting experience of the last $A N C$ ' (FG $G_{2}$ woman $X_{10}$ ).

The PCV test that you did for us, the first test was $36 \%$, by my second test, it reduced to $32 \%$ so you encouraged me to eat more of vegetables I picked it up, I started eating vegetables and fruits and the last PCV was $39 \%$ such that even after delivery, my PCV was still 36\%. I didn't have that awareness during my previous pregnancies but this one I got that awareness so this interests me most' (FG4, woman $A_{6}$ ).

Motivations for ANC attendance. The quest for wellbeing through the care received at ANC was reported by participants as the major driving force that motivated them to attend ANC. This care is rendered through physical assessment and laboratory examinations of the pregnant women and their unborn babies. The education on personal and environmental hygiene they acquired during health talks at ANC also generated strong motivation for ANC attendance. When asked why they decided to register and attend antenatal clinic, the women responded thus:

'I come to antenatal clinic to know the general well-being of myself and the baby I am having' $\left(F G_{2}\right.$ women $\left.X_{10}\right)$. 'I come to antenatal clinic to know how I and my baby are faring and to identify and handle any problem early $\left(F G_{4}\right.$ woman $\left.A_{10}\right)$. 'I attend ANC because they have specialists who check to know the position of my baby and to receive specialist care, like in my own case, my BP kept rising till they delivered me before the time. If I had not come for ANC maybe something else could have happened.' $\left(F G_{3}\right.$ woman $\left.C_{8}\right)$.

'As for me the lectures (health talk) given to us on every ANC day has helped a lot. I feel relieved because something I don't understand about myself, when I come here, I asked questions' ( $F G_{3}$ woman $\left.C_{6}\right)$. 'It is compulsory for every pregnant woman to attend antenatal' $\left(F G_{1}\right.$ woman $\left.P_{4}\right)$. 'The care I received in the ANC made my baby to be okay, strong and balanced' $\left(F G_{l}\right.$, woman $\left.P_{7}\right)$.

Factors that were reported to have influenced the choice of ANC to register and access services included: testimonies of good care rendered by a given centre, proximity to residence, cost of care, availability of specialists and equipment to deliver adequate care. Primigravid women appear to mostly rely on the testimonies and experiences of multiparous women in deciding where to register for ANC. They said:

'I used to go to private hospitals before but when I heard how good they take care of pregnant women here, I tried it and I liked it' $\left(F G_{1}\right.$ woman $\left.P_{14}\right)$. 'It is in this hospital (Central Hospital) that my mother gave birth to all her eight children without any complain and my first baby was also born here because they have qualified doctors and nurses' ( $F G_{3}$ woman $C_{11}$.

'Here (Central Hospital), they have equipment and facilities provided by government and it is affordable, there doctors are well trained; even my mum gave birth to me here and I also came here on their recommendations' ( $F G_{2}$ woman $X_{10}$ ).

Attending ANC has shown to be of great help to the study participants as they shared their benefits and experiences. They expressed good understanding of the health talk that they received and how it helped them to know how to detect early signs of danger and to seek prompt medical attention to avert complications.

'Attending antenatal has really helped me a lot, because this is my first baby. I was eager (anxious) of so many things but attending antenatal clinic they taught me how to take care of my baby after delivery and myself' $\left(F G_{3}\right.$ woman $C_{12}$ ). 
'In fact, I look up to ANC days with joy because of the spiritual moments and encouragement we get which prepared us spiritually. It also helped to know how well your baby is doing from their assessment' ( $F G_{2}$ woman $X_{1}$ ).

\section{Suggestions for improvement in ANC} services. The women generally commended the staff for their efforts in caring for them, but observed that some services they were supposed to benefit from were not being done. It was observed that pregnant women were not weighed during clinic visits, urine tests are only done at booking and body temperature was not always taken. These examinations they considered to be very vital in monitoring their health and progress but which were not being done.
Other suggestions revolved around staff attitude and registration procedures. They observed that staff were sometimes harsh and unfriendly to them which often add to their pains. The process of registration (ANC booking) was observed to be cumbersome and stressful. There is the need for a side laboratory in the ANC clinic for pregnant women to ease their stress, especially queuing for long to be attended to in central laboratories. In addition, they suggested that more doctors and nurses should be employed to ease the pressure on the few ones and that it will also improve staff-client relationship.

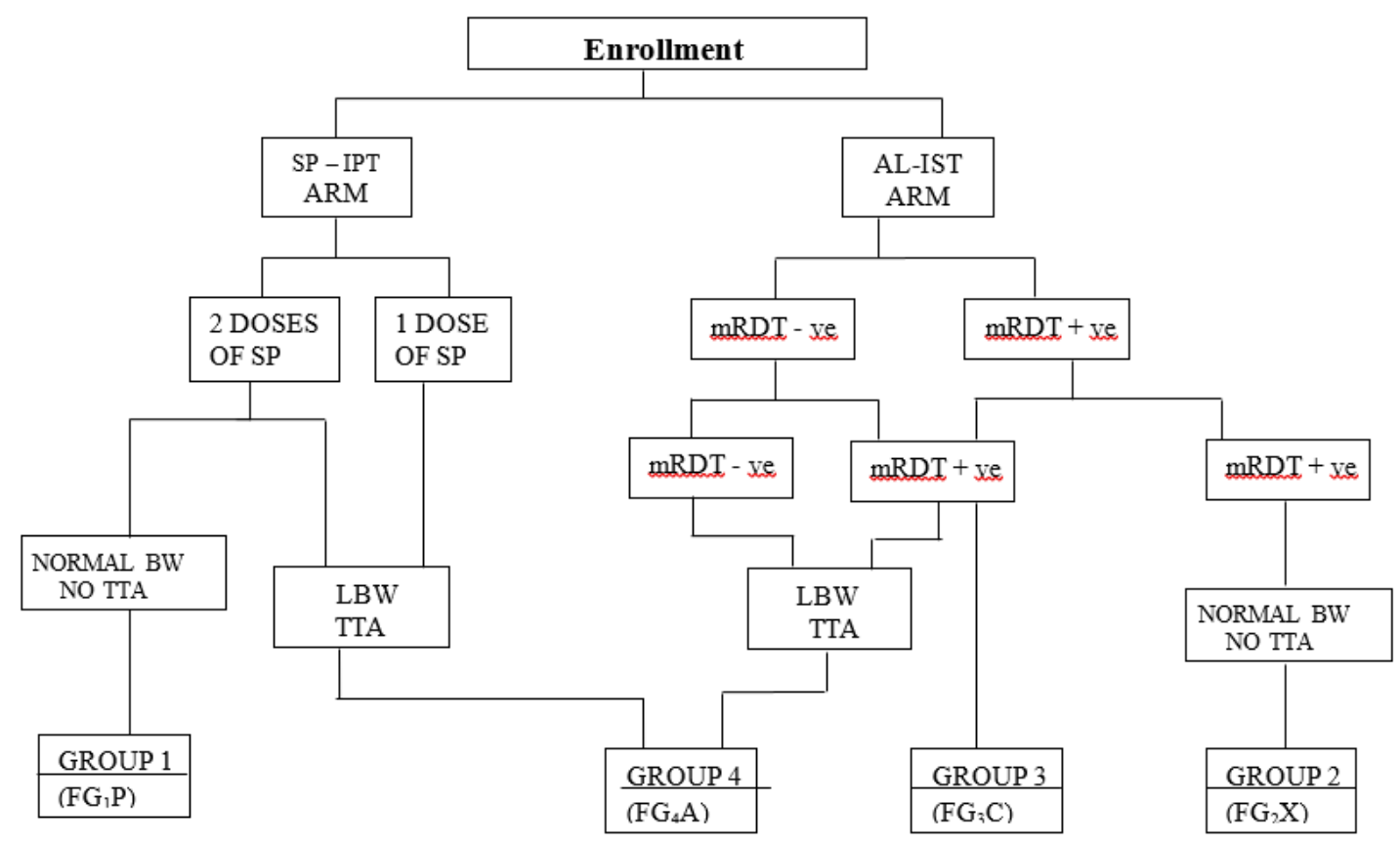

LBW $=$ Low birth weight, TTA $=$ Third trimester anaemia, $\mathrm{BW}=$ Birth weight

Figure 1: Recruitment strategy of the study participants

Table 1: Profile of study participants for the focused group discussions

\begin{tabular}{cccccc}
\hline Group & Symbol & Client Category & No. of women & Age range & Primary outcome \\
\hline 1 & $\mathrm{FG}_{1} \mathrm{P}$ & SP-IPTp (2 doses) & 4 & $27-34 \mathrm{yrs}$ & NLPA and NBW \\
2 & $\mathrm{FG}_{2} \mathrm{X}$ & AL-ISTp (mRDT -Ve) & 9 & $26-42 \mathrm{yrs}$ & NLPA and NBW \\
3 & $\mathrm{FG}_{3} \mathrm{C}$ & AL-ISTp (mRDT +Ve) & 8 & $27-42 \mathrm{yrs}$ & Nonspecific Outcome \\
4 & $\mathrm{FG}_{4} \mathrm{~A}$ & SP-IPTp \& AL-ISTp & 10 & $25-41 \mathrm{yrs}$ & LPA \& or LBW \\
\hline
\end{tabular}




\section{DISCUSSION}

There was little difference in the test procedure between the two treatment arms as all enrolled women underwent repeated prick of finger tips for blood tests. Blood samples were obtained for the malaria test from the same prick for PCV test. This made the emerged themes to be similar across the treatment arms and all study groups sharing similar perceptions and experiences. The wellbeing of the mothers and babies was the most motivating factors for ANC attendance. This was found to be reinforced by previous experiences and/or testimonies of how ANC had helped averted serious dangers and solved pregnancy complications in the past. Knowing their wellbeing and that of their unborn babies was quite reassuring of a safe gestation and reinforces the hope of safe and live birth. This understanding drives away fear and doubts and builds up a positive image and emotions that compliments the physical health. This view is supported by finding from similar studies [16, 18] where most antenatal women discussants in a similar study reported that wanting to know if there were any problems for themselves or for the baby with specific concerns about avoiding adverse events, (in particular losing the baby) as their driving force to attendance at ANCs.

The study revealed that some women migrate from clinic to clinic during pregnancy in search of better services. Adequate care and good attitude of staff to clients was the primary driving force in women choosing which clinic to register for ANC. Secondary factors included proximity and cost of care. This suggests that women may be willing to pay for good, friendly and quality care. This was not a general consensus as some women specifically considered cheap cost of care (in government hospitals) as their primary motivation in deciding where to register for ANC.

Many women showed good understanding that this was a project and that certain procedures had to be followed and so were willingly accepting the explanation we gave them during the tests. This was contrary to the view and fear we had earlier entertained before commencement of the tests. Many women in the IST group were happy when they were told that they had no malaria and so did not need any drug. This is contrary to the feeling of some women in the IPT group who preferred to have also been tested for malaria.

The women across treatment arms reported that they felt very happy that their PCV was monitored, this made them make efforts to boost it whenever it was found to be low. Women reported that the pain of hand pricking associated with the IST practice was bearable since it was for their own good. Though being involved in a trial per se could have a positive effect on perceptions on the services received [19], the positive perceptions reported by the women in this study are not viewed to be merely based on their involvement in the trial but their reported confidence in the derivable benefits of the IST program.

This acceptance may result from the trust that these women have in the investigators may be because they were told the result of the tests immediately and its implication on their health and the advice given to them. Some of the discussants said this was contrary to how they were treated in the central laboratories where it takes days for them to get the result for same tests and those results are never explained to them. They only get to guess the result when the doctor sees it and prescribed drug for them to take. For example, some women particularly appreciated their participation in the trial because the test results were fully explained to them and this subsequently helped them a lot.

Many of the study participants (cutting across both arms) expressed their preference for the IST strategy despite the pains involved in finger pricking. Some women who had shown preference for IPT changed their mind when the IPT and IST principles were 
explained in detail to them, reporting IST as the most ideal and acceptable strategy, but observed that the RDT kids must demonstrate sensitivity to detect low plasmodium parasites.

Though it may not be easy to translate the experiences of participants during the study under trial conditions to the experiences of implementation within the routine ANC for clinic staff because of increased workload [16], it is observed that the only difference should IST be introduced to replace the SP-IPT would be the need for regular RDTs to be carried out and so an extra hand and additional time will be required to overcome the burden on clinic staff. All other investigations and services remain a routine they are accustomed to. It is reported that malaria-related community lead interventions require deliberate and constant community engagement activities [20]. This focus group discussion is part of such community engagement that open the community to all available health interventions for them to choose what best meet their health needs which when implemented as a policy will have high uptake by the community.

The main challenges envisaged in the implementation of IST is in the area of staffing and stock out of RDT kits and antimalarial drugs. In smaller clinics with fewer staff, it implies that women accessing the service may wait longer than before to be attended to. Frequent stock out of RDT kits and antimalarial drugs will impede the effective implementation of an IST programme. These challenges can be overcome if a revolving fund is established for the programme and staff are assigned to specifically handle the tests aspect to be incorporated into the time of scheduled services of the ANC. Furthermore, increased enrolment into the National Health Insurance Scheme (NHIS) means that the problem of cost will eventually become a thing of the past as all maternal health services would be delivered without the burden of out-of-pocket payment. These combined with effective monitoring and supervision of programme implementation will be critical to the success of any maternal health intervention in general and IST in particular.

The treatment regimen for Coartem ${ }^{\circledR}$ the drug used for IST arm in this study is one tablet in the morning and one in the evening for three days while SP for IPT is single dose. IST strategy will not have any problem of adherence because it will be treatment based on proof of existing presence of parasites in the system which is reported by study participants as a motivation for taking drugs during pregnancy. This has been reported to be a motivation even for women who do not like taking drugs, whether or not they exhibit symptoms of malaria. In addition, adherence can be enhanced through appropriate education by the providers [21-23]. These group discussions enabled participants to give an account of the various ways in which the interventions impacted on them which cannot be measured and quantified by the tests.

\section{Conclusion}

This study revealed that pregnant women in these settings showed higher preference for IST compared to IPT as a strategy for the control of malaria in pregnancy. The perceived benefit of knowing their health status with respect to malaria and the fear of taking drugs without indication during pregnancy (recalling from past experiences of doing so) were found to be the most influential factors to their choice of IST as a strategy. Following the high uptake of ANC services by pregnant women, IST promises to be a more successful malaria control programme for pregnant women when made free and effectively implemented.

\section{REFERENCES}

1. Federal Republic of Nigeria (2015): Nigeria Malaria Indicator Survey (NMIS) Report

2. Menendez C, D’Alessandro U, ter Kuile FO (2007): Reducing the burden of malaria in pregnancy by preventive strategies. Lancet Infect Dis, 7:126-135. 
D.W. Dayom et al. / J. Pharmacy \& Bioresources 17(2), 142-152 (2020)

3. ter Kuile FO, van Eijk AM, Filler SJ (2007). Effect of sulfadoxine-pyrimethamine resistance on the efficacy of intermittent preventive therapy for malaria control during pregnancy: a systematic review. JAMA, 297:2603-2616.

4. Grietens KP, Gies S, Coulibaly SO, Ky C, Somda J, Toomer E, Muela Ribera J, D'Alessandro U (2010). Bottlenecks for high coverage of intermittent preventive treatment in pregnancy: The case of adolescent pregnancies in rural Burkina Faso. PLoS One, 5:e12013.

5. Uddenfeldt WU, Hastings I, Bergstrom S, Massawe S, Lipingu C, Brabin BJ (2007). Increased postpartum blood loss in pregnancies associated with placental malaria. Int J Gynaecol Obstet, 96:171-175.

6. Apinjoh, T. and Agbor, L. (2017): Evidence of plasmodium falciparum resistance to SulphadoxinePyrimethamine (SP) in pregnant women along the slope of Mount Cameroon. BMJ Glob Health; 2 (Suppl 2):A1-A67

7. Newman RD, Parise ME, Slutsker L, Nahlen B, Steketee RW (2003) Safety, efficacy and determinants of effectiveness of antimalarial drugs during pregnancy: Implications for prevention programmes in Plasmodium falciparum-endemic subSaharan Africa. Tropical Medicine \& International Health 8: 488-506.

8. Anthony MP, Jeremy N Burrows, Stephan Duparc, Joerg JMoehrle and Timothy NC Wells (2012). The global pipeline of new medicines for the control and elimination of malaria. Malaria Journal, 11:316 http://www.malariajournal.com/content/11/1/316

9. Ouma OP (2012). Improving drug regimens and implementation strategies for malaria prevention in pregnant women in Western Kenya: A dissertation, chapter 8. http://dare.uva.nl/document/357670. Accessed 25/10/2012

10. Sicuri E, Fernandes S, Macete E, González R, Mombo-Ngoma G, Massougbodgi A, et al. (2015): Economic Evaluation of an Alternative Drug to Sulfadoxine-Pyrimethamine as Intermittent Preventive Treatment of Malaria in Pregnancy. PLoS ONE 10(4): e0125072. doi:10.1371/journal. pone. 0125072

11. Tagbor H, Bruce J, Agbo M, Greenwood B, Chandramohan D (2010) Intermittent Screening and Treatment versus Intermittent Preventive Treatment of Malaria in Pregnancy: A Randomized Controlled Non-Inferiority Trial. PLOS ONE 5(12): e14425. doi:10.1371/journal.pone.0014425
12. O’Meara, WP, Brian Greenwood, J N Mangeni R Steketee (2010). Changes in the burden of malaria in sub-Saharan Africa Lancet Infect Dis; 10: 545-55

13. World Health Organization (2018): Regional and global trends in the burden of malaria. World malaria report.

14. Baltzell KA, Maglior A., Bangu K,. Mngadi N., Prach LM, Whittemore B., Ntshalintshali N., Saini M., Dlamini N., Kunene S. and Hsiang MS (2019): "We were afraid of the lion that has roared next to us"; community response to reactive focal mass drug administration for malaria in Eswatini (formerly Swaziland). Malaria Journal, 18:238

15. Gericke CA, Kurowwski C, Ranson MK, Mills A (2005). Intervention complexity-a Conceptual framework to inform priority setting in health. Bull World Health Organ, 83:285-293.

16. Smith LA, Jones C, Adjei RO, Antwi GD, Afrah NA, Greenwood B, Chandramohan D, Tagbor H, and Webster J (2010). Intermittent screening and treatment versus intermittent preventive treatment of malaria in pregnancy: user acceptability. Malaria Journal, 9:18 doi:10.1186/1475-2875-9-18

17. Pell C, Arantza M, Samuel C, Abraham H, Tagbor H and Robert P (2014). The acceptability of intermittent screening and treatment versus intermittent preventive treatment during pregnancy: results from a qualitative study in Northern Ghana. Malaria Journal, 13:432

18. Mrisho M, Obrist B, Schellenberg JA, Haws RA, Mushi AK, Mshinda H, Tanner M, Schellenberg D. (2009). The use of antenatal and postnatal care: perspectives and experiences of women and health care providers in rural southern Tanzania. BMC Pregnancy Childbirth, 9:10.

19. Mfutso-Bengo J, Ndebele P, Jumbe V, Mkunthi M, Masiye F, Molyneux S, Molyneux M (2008). Why do individuals agree to enrol in clinical trials? A qualitative study of health research participation in Blantyre, Malawi. Malawi Med J, 20:37-41.

20. Kaunda-Khangamwa BN, Berg H, McCann RS, Kabaghe A, Takken W, Phiri K, Vugt MV and Manda-Taylor L (2019): The role of health animators in malaria Control: a qualitative study of the Health Animator (HA) approach within the Majete Malaria project (MMP) in Chikwawa District, Malawi. BMC Health Services Research, 19:478

21. Ajayi IO, Browne EN, Garshong B, Bateganya F, Yusuf B, Agyei-Baffour P, Doamekpor L, Balyeku A, Munguti K, Cousens S, Pagnoni F (2008): Feasibility and acceptability of artemisinin-based combination 
therapy for the home management of malaria in four African sites. Malaria Journal, 7:6.

22. Beer N, Ali AS, Rotllant G, Abass AK, Omari RS, Al-mafazy AW, Bjorkman A, Kallander K (2009):

Adherence to artesunate-amodiaquine combination therapy for uncomplicated malaria in children in Zanzibar, Tanzania. Trop Med Int Health, 14:766774.
23. Tagbor H, Bruce J, Browne E, Randall A, Greenwood B, Chandramohan D (2006): Efficacy, Safety and tolerability of amodiaquine and sulphadoxine/pyrimethamine used singly or in combination for the treatment of malaria in pregnancy. Lancet, 368:1349-1356. 